\title{
Deictic gestures and symbolic gestures produced by adults in an experimental context: Hand shapes and hand preferences
}

\author{
Hélène Cochet ${ }^{1}$ and Jacques Vauclair ${ }^{2}$ \\ ${ }^{1}$ School of Psychology \& Neuroscience, University of St Andrews, Fife, \\ UK \\ ${ }^{2}$ Department of Psychology, Aix-Marseille University, \\ Aix-en-Provence, France
}

\begin{abstract}
The objective of this study was to gain new insights into the processes underlying gestural communication in adults by examining hand shapes and hand preference patterns associated with different types of gestures. Several communicative situations eliciting pointing gestures and symbolic gestures were presented to 81 participants in an experimental context. Results have highlighted some differences in hand shapes depending on the function of pointing: contrary to results reported in children, the proportion of index-finger gestures was higher in imperative situations than in declarative situations. The distance between the gesturer and the referent was also found to influence hand shapes, proximal pointing being more frequently associated with index-finger gestures than distal pointing. The comparison of hand preferences revealed a greater right-sided asymmetry for declarative pointing than for non-communicative activities, whereas there was no difference between imperative pointing and non-communicative activities, or between symbolic gestures and non-communicative activities. The present study thus sheds some light on the features and the roles of communicative gestures in adults. Results are discussed in relation to the ontogenetic and phylogenetic origins of communication.
\end{abstract}

Keywords: Pointing gestures; Symbolic gestures; Hand shapes; Hand preference.

Systematic observations of adults' gestures have highlighted the diversity of the human gestural repertoire in terms of communicative function, hand

Address correspondence to: Hélène Cochet, Centre for Social Learning \& Cognitive Evolution, School of Psychology \& Neuroscience, University of St Andrews, Fife KY16 9JP, Scotland, UK. E-mail: hc42@st-andrews.ac.uk

This research was supported by a French National Research Agency (ANR) grant, reference ANR-08-BLAN-0011_01 and by a postdoctoral research grant from the Fyssen foundation.

(C) 2013 Taylor \& Francis 
shape (including palm orientation, and movement and position of the hand in the gesture space), and hand preference (e.g., Kendon, 2004; Kita, Condappa, \& Mohr, 2007). Yet the study of non-verbal communicative behaviours in adults has been subject to some unwarranted assumptions, in part because examining the characteristics of different gestures requires a controlled physical environment, without actually constraining the participants' responses. In particular, few studies have focused on gestures other than co-speech gestures (e.g., McNeill, 2000).

In the present study, through experimental situations allowing participants to produce spontaneous responses, we investigated how the form and the laterality of gestures vary with their functions by considering two main functional categories: symbolic gestures (also referred to as conventional gestures, or emblems; McNeill, 1992) and deictic gestures (e.g., Crais, DayDouglas, \& Cox-Campbell, 2004; Liszkowski, 2008). By finding out to what extent features like hand shape and hand preference convey some information that complements the communicative message, our objective was to improve the understanding of the role and the origin of these gestures in adulthood.

\section{DEICTIC GESTURES AND SYMBOLIC GESTURES}

First, deictic gestures are produced to direct a recipient's attention towards a specific referent in the proximal or distal environment. The pointing gesture is the main representative of this category and it can fulfil several communicative functions. Beyond cross-cultural variations of gestures that have been well documented (e.g., Enfield, 2001; Kita, 2009), hand shapes associated with pointing gestures also vary within cultural groups according to the communicative context and to the nature of the information being conveyed (e.g., Enfield, Kita, \& De Ruiter, 2007). Thus adults frequently use index-finger pointing to disambiguate referential situations whereas wholehand pointing and thumb pointing are mostly produced in situations requiring little precision (e.g., Wilkins, 2003). Palm orientation is also influenced by the importance of the referent to the ongoing discourse: Kendon and Versante (2003) showed that referents in the centre of discourse focus are more likely to be indicated by the index finger with the palm down rather than with the palm vertical.

In the present study we have considered several different functions to broadly explore the form of pointing gestures, using a distinction that was initially made in infants studies: imperative pointing has been described as a request for a desired object or an action on that object, declarative expressive pointing aims at sharing some interest with the recipient in a specific object or event, and declarative informative pointing is produced to help a communicative partner by providing him/her needed information (e.g., Bates, Camaioni, \& Volterra, 1975; Camaioni, 1997; Tomasello, Carpenter, \& 
Liszkowski, 2007). In children, declarative and imperative functions seem to be mainly associated with index-finger pointing and whole-hand pointing, respectively (Cochet \& Vauclair, 2010b). Moreover, index-finger declarative pointing was shown to be more frequently accompanied by vocalisations and gaze alternation between the referent and the communicative partner than whole-hand pointing, and was therefore suggested to be directly related to the development of intention-reading skills (Cochet \& Vauclair, 2010a; Liszkowski \& Tomasello, 2011). This hypothesis is in agreement with the increasing proportion of index-finger pointing reported as children grow old in declarative situations. By contrast, the form of imperative gestures was not found to vary in the course of development (between 1 and 3 years of age; Cochet \& Vauclair, 2010b), indicating that the use of index-finger or wholehand pointing in imperative situations may characterise one's own gestural repertoire. In adults, although the above-mentioned studies have emphasised the relationship between the form of pointing gestures and contextual features, some individuals may also develop a specific repertoire regardless of the situation.

Other variables may influence the form of pointing gestures, such as the distance between the referent and the communicative partners, although to our knowledge there have been no systematic studies on this question. Franco and Butterworth (1996) did vary this distance in declarativereferential and imperative-instrumental conditions of communication, but could not draw any firm conclusion because context and distance were confounded. The distance from the gesturer may influence hand shapes because it is usually linked to the degree of precision required to identify the referent: points towards distant objects (or objects that are not visible) are more likely to be characterised by whole-hand gestures as it might not be difficult or essential to identify them accurately. By contrast, referents that are close to the gesturer may necessitate a higher level of individuation, and might therefore be associated with index-finger pointing. However, one could also argue that a precise gesture might not be needed when the location of the referent is obvious. Thus, comparing proximal and distal situations can help determine the precise effect of this distance variable on the form of pointing gestures.

Second, symbolic gestures represent directly a specific object or action (e.g., nodding the head, waving "goodbye"), based on conventionality or habit within the same cultural group. Any variation in the form of symbolic gestures could therefore alter the meaning conveyed, which is why it may be less subject to variations than the form of pointing gestures. However, variables such as the amount of information shared by the gesturer and the addressee (i.e., the "common ground") have been shown to have an effect on the form of some representational gestures used to depict actions (Gerwing \& Bavelas, 2004). Moreover, it is still unclear to what extent a given situation 
can evoke different forms of symbolic gestures (Parrill, 2008), and this question has mainly been examined in qualitative studies so far (e.g., Morris, Collett, Marsh, \& O’Shaughnessy, 1979).

\section{HAND PREFERENCES}

Several studies have investigated the asymmetry of pointing gestures and symbolic gestures produced by young children (e.g., Bates, O'Connell, Vaid, Sledge, \& Oakes, 1986; Vauclair \& Cochet, 2012), whereas the analysis of hand preference patterns for gestures in adults has mainly concerned cospeech gestures (e.g., Dalby, Gibson, Grossi, \& Schneider, 1980; Kimura, 1973; Saucier \& Elias, 2001). However, most co-speech gestures are used to give some emphasis and rhythm to the discourse and may not necessarily involve any communicative intention (see McNeill, 2005), which is why we chose to focus exclusively on hand preference associated with deictic gestures and symbolic gestures in the present study. In order to be certain about the communicative and intentional nature of the gestures produced, we set up situations in which the latter were necessary to convey a specific message.

Thus one of our objectives was to gain some insights into hemispheric specialisation for communicative behaviours in adults. In this perspective, Kimura (1973) showed that the right-sided asymmetry for gestures was related to hemispheric dominance for language, and more recently several neuro-imaging studies have shown that speech and gestures were controlled by similar networks in the left cerebral hemisphere (e.g., Gentilucci \& Dalla Volta, 2008; Xu, Gannon, Emmorey, Smith, \& Braun, 2009). However, these studies focused on a great variety of gestures, from free co-speech movements defined as "any motion of the limb which did not result in touching of the body or coming to rest" (Kimura, 1973, p. 46) to pantomimes (e.g., Xu et al., 2009). Therefore we still have to determine whether the relationship between gestures and language involves some gestures more than others, or whether it stands for every kind of movements associated with a communicative function. In this study we thus used the asymmetry of pointing and symbolic gestures as an indicator, although indirect, of the relationship between gesture and language.

In young children a particularly strong right-sided bias has been reported for declarative informative pointing compared to other communicative functions of pointing (Cochet \& Vauclair, 2010b). We might expect to observe the same result in the present study; however, the use of similar indices in young children and in adults has revealed different patterns of hand preference (e.g., Cochet \& Vauclair, 2012). More specifically, while children between approximately 1 and 3 years of age are more right-handed for communicative gestures than for manipulative activities (e.g., Bates et al., 1986; Vauclair \& Imbault, 2009), there is no difference in the degree of 
asymmetry for these different activities in adults (Cochet \& Vauclair, 2012). Therefore we sought to assess hand preferences associated with the different functions of pointing gestures and symbolic gestures, as well as handedness for manipulative activities for comparative purposes. The experimental context of this study allowed us to control the laterality variable, since the respective spatial localisations of the gesturer, the referent and the recipient can influence hand choice (e.g. Butterworth, Franco, McKenzie, Graupner, \& Todd, 2002).

Finally, the comparison of hand preferences for different communicative activities can shed some light on the hypothetical scenarios that led to language and to human hemispheric specialisation for language, at both the ontogenetic and phylogenetic levels. There is a close relationship in young children between the production of pointing gestures and language development (e.g., Colonnesi, Stams, Koster, \& Noom, 2010). Declarative pointing in particular, because it seems to involve the understanding of referential intentions (e.g., Liszkowski, Carpenter, Henning, Striano, \& Tomasello, 2004; Liszkowski, Carpenter, Striano, \& Tomasello, 2006), has been suggested to be the precursor of verbal language (e.g., in 12-month-old infants: Liszkowski \& Tomasello, 2011). The emergence of referential skills through the use of pointing gestures may also have been crucial among our hominin ancestors. By contrast, some researchers have argued for a key role of symbolic gestures in the evolution of language (e.g., Gentilucci, Dalla Volta, \& Gianelli, 2008; Kendon, 2009). Comparing the strength of the rightsided bias for the different functions of pointing and for symbolic gestures in the present study might allow us to favour one hypothesis over the other. Although it gives only indirect evidence, the analysis of gestures produced by adults may thus supplement the studies in young children and in nonhuman primates, and help in deciphering the ontogenetic and phylogenetic origins of communication and manual asymmetries in our species (e.g., Cochet \& Byrne, 2013).

\section{OBJECTIVES OF THE PRESENT STUDY AND HYPOTHESES}

In the present study we investigated the relationship between the form, the function, and the laterality of gestures produced by young adults. We sought to determine whether the variations in hand shapes and in hand preference patterns depending on contextual features can help to uncover the roles of pointing gestures and symbolic gestures in communication. We set up experimental communicative situations to elicit several gestures in order to facilitate the comparison between participants and thus identify more easily the variables likely to influence hand shapes and hand preferences.

First, we aimed to investigate the differences between the three functions of pointing gestures that have already been studied in children (Tomasello 
et al., 2007). We expected imperative gestures to be more frequently associated with whole-hand pointing, and declarative gestures (both expressive and informative) to be more frequently associated with indexfinger pointing (e.g., Cochet \& Vauclair, 2010b; Franco \& Butterworth, 1996). In addition we distinguished between proximal and distal situations to determine the influence of the distance between the gesturer and the referent on hand shapes. Distal pointing is generally used for large-sized referents or rather vague targets that may even not be visible, thus requiring less precision than proximal pointing. Therefore our hypothesis was that distal pointing would be more frequently characterised by whole-hand gestures, while proximal pointing would be more frequently characterised by indexfinger gestures.

Second, we intended to study the production of symbolic gestures in comparable situations across participants who have all been living in a French cultural environment and who were of similar age. Although each situation was designed to elicit the same gesture (described as the "target gesture"), we aimed to identify the variations in the form of symbolic gestures by assessing the proportion of gestures that would not match the target description. Such an analysis for each different situation was expected to be a first step towards understanding the causes of these variations.

Lastly we sought to study hand preference patterns for pointing gestures and symbolic gestures in an attempt to fill a gap in the literature on manual asymmetries in adult populations. Comparing the asymmetry of different communicative gestures and that of manipulative activities (measured with a self-report questionnaire) may allow us to determine whether the lefthemispheric specialisation, as reflected by right-handedness, involves more specifically one category of activities. Given the relationship between gesture and language (e.g., Gentilucci \& Dalla Volta, 2008), we expect symbolic gestures and pointing gestures (declarative pointing in particular) to be more right-handed than manipulative activities. However, we may also observe a moderate correlation in the strength of the right-sided bias for communicative and manipulative activities (Cochet \& Vauclair, 2012).

\section{METHOD}

\section{Participants}

A total of 81 French university students between 17 and 27 years of age ( $M=19.3$ years; $S D=1.6$ years), including 37 men and 44 women, were tested individually in a university experimental room. Participants signed an informed consent form and they were informed only at the end of the experiment that we recorded hand preferences of gestures. All sessions were videotaped. 


\section{Procedure}

Participants were seated at a rectangular table, with the experimenter sitting opposite them reading out loud the different communicative scenarios. A total of 13 scenarios were proposed in a randomised order across participants, referring to situations in which gestures proved to be a natural and efficient communicative modality. Six scenarios were designed to elicit pointing gestures serving different functions (imperative, declarative expressive, and declarative informative functions), with either a proximal or distal referent (proximal referents were located more than two metres away from the gesturer), and seven scenarios were designed to elicit symbolic gestures (see Appendix for a full description of the scenarios and corresponding gestures).

Participants were asked to listen carefully and imagine themselves in each communicative situation before producing an appropriate communicative response, directed towards the experimenter. Participants were asked to produce what they would spontaneously do in the different contexts, and the experimenter did not specify that they had to use gestures. Moreover, the latter did not produce any gesture that could influence the participant's response. However, in the few cases in which participants did not produce any gesture the experimenter asked them whether there might be a gesture that they could use in such a situation. If the participants still did not produce any manual gesture, the experimenter moved on to the following scenario.

In order to avoid any positional bias participants were asked to place their hands on the table between each scenario, on two symmetrical stickers that had been positioned $25 \mathrm{~cm}$ away from the edge of the table. After listening to the communicative scenarios, participants filled in a 13-item hand preference questionnaire based on the Edinburgh Handedness Inventory (Oldfield, 1971) which provided a measure of hand preference for manipulative activities. Participants were asked to indicate for each activity (e.g., using a spoon) whether they preferred to use the left or the right hand, or whether they could use both hands equally (coded as bimanual).

\section{Data analyses}

Form of the gesture. We noted hand shapes associated with each pointing gesture. More specifically, we differentiated index-finger pointing (defined as the extension of the index with the other fingers tightly curled) from wholehand pointing (defined as the extension of all fingers). When the index finger was extended with the other fingers only lightly curled, hand shape was coded as intermediate. We also recorded palm orientation, distinguishing between palm down, palm lateral, and palm up positions. 
Regarding symbolic gestures, we noted hand shapes when they did not correspond to the reference description (see Appendix). These gestures were included in the analyses of hand preference provided they fulfilled the same communicative function as the target gesture.

Hand preference. An individual handedness index score $(\mathrm{HI})$ was calculated for each participant using the formula $(\mathrm{R}-\mathrm{L}) /(\mathrm{R}+\mathrm{L}+\mathrm{B})$, where $\mathrm{R}$ and $\mathrm{L}$ stand for the total right- and left-hand responses, and $\mathrm{B}$ stands for bimanual responses. The HI values lay along a continuum from -1 to 1 , with the sign indicating the direction of hand preference and the absolute value characterising the strength of hand preference. HI scores were calculated for communicative gestures and manipulative activities. We also conducted analyses by distinguishing, within the category of communicative gestures, between symbolic gestures and the different functions of pointing gestures. From the HIs, participants were characterised as right-handers $(\mathrm{HI} \geq 0.50)$, left-handers $(\mathrm{HI} \leq-0.50)$ or non-lateralised $(-0.50<\mathrm{HI}<0.50)$. We included bimanual responses in the calculation of handedness indices (whereas researchers do not always do so: e.g., Cochet \& Vauclair, 2010a; Corbetta \& Thelen, 1999) to provide more accurate and therefore more representative measures of individuals' handedness (e.g., Jacquet, Esseily, Rider, \& Fagard, 2012), as bimanual responses were relatively numerous in the present study (see the results section).

\section{RESULTS}

In total, participants produced between 6 and 13 communicative manual gestures $(M=11.0 ; S D=1.3)$. More specifically, they produced between 4 and 6 pointing gestures $(M=5.6 ; S D=0.6)$ and between 2 and 7 symbolic gestures $(M=5.4 ; S D=1.1)$. Simultaneously to the production of gestures, the vast majority of participants complemented their message with verbal utterances. The speech-gestures interactions deserve a fully fledged investigation, and will be the subject of another paper.

\section{Form of the gestures}

Pointing gestures. Participants produced 5 bimanual gestures out of a total of 444 gestures (i.e., approximately $1.1 \%$ ), which were included in the analyses, as the form was identical for both hands. We first focused on the percentages of index-finger and whole-hand pointing observed in each situation (see Table 1). The majority of declarative expressive gestures $(80.3 \%)$ were associated with index-finger pointing but, contrary to our hypotheses, imperative gestures were mostly characterised by index-finger pointing $(91.2 \%$ of imperative 
TABLE 1

Percentages of index-finger and whole-hand pointing gestures produced (on the total number of gestures produced) depending on the function of the gesture and on the distance between the gesturer and the referent. Values were rounded to the nearest integer

\begin{tabular}{lcccc}
\hline & $\begin{array}{c}\text { Total number of } \\
\text { gestures produced }\end{array}$ & $\begin{array}{c}\text { Index-finger } \\
\text { pointing }\end{array}$ & $\begin{array}{c}\text { Whole-hand } \\
\text { pointing }\end{array}$ & $\begin{array}{c}\text { Intermediate hand } \\
\text { shape }\end{array}$ \\
\hline Imperative proximal & 50 & $89 \%$ & $9 \%$ & $2 \%$ \\
Imperative distal & 80 & $93 \%$ & $6 \%$ & $1 \%$ \\
Expressive proximal & 80 & $92 \%$ & $4 \%$ & $4 \%$ \\
Expressive distal & 81 & $68 \%$ & $27 \%$ & $5 \%$ \\
Informative proximal & 72 & $63 \%$ & $33 \%$ & $4 \%$ \\
Informative distal & 81 & $42 \%$ & $56 \%$ & $2 \%$ \\
\hline
\end{tabular}

gestures) and only approximately half of declarative informative gestures $(51.6 \%)$ were associated with index-finger pointing.

For the following analyses we excluded intermediate hand shapes as the number of gestures was too low in this category (see Table 1). Chi-square tests revealed that imperative pointing was more frequently associated with index-finger gestures than declarative expressive pointing, $\chi^{2}=5.07, d f=1$, $p<.05$ and declarative informative pointing, $\chi^{2}=53.5, d f=1, p<.001$. Moreover, declarative expressive pointing was more frequently associated with index-finger gestures than declarative informative pointing, $\chi^{2}=32.9$, $d f=1, p<.001$.

Second, we also took into account the distance between the gesturer and the referent. Proximal pointing was more frequently associated with indexfinger gestures than distal pointing in declarative expressive and declarative informative situations, $\chi^{2}=17.5, d f=1, p<.001$ and $\chi^{2}=7.3, d f=1, p<.01$, respectively. By contrast, in imperative situations there was no significant difference in the proportion of index-finger gestures between proximal and distal pointing, $\chi^{2}=0.36, d f=1, n s$.

Third, we investigated palm orientation associated with pointing gestures (see Table 2), and more precisely with whole-hand pointing since indexfinger pointing was almost exclusively produced with a palm down position or with an intermediate position between palm down and palm lateral. Declarative expressive pointing was more frequently associated with palm down gestures and declarative informative pointing with palm lateral and palm up gestures, $\chi^{2}=51.46, d f=2, p<.001$. Statistical analyses could not be performed for imperative pointing due to the small number of gestures produced. Moreover, the combined effect of the function and the distance on palm orientation could only be described with qualitative analyses, as the number of gestures was too limited in some categories. Declarative proximal gestures (both expressive and informative gestures) were mostly 
TABLE 2

Percentages of palm orientation for whole-hand pointing gestures depending on the communicative situation. Values were rounded to the nearest integer

\begin{tabular}{lcccc}
\hline & Total number of gestures produced & Palm lateral & Palm down & Palm up \\
\hline Imperative proximal & 5 & $60 \%$ & $40 \%$ & - \\
Imperative distal & 5 & - & $100 \%$ & - \\
Expressive proximal & 3 & - & $33 \%$ & $67 \%$ \\
Expressive distal & 22 & $9 \%$ & $77 \%$ & $14 \%$ \\
Informative proximal & 24 & $99 \%$ & $8 \%$ & $63 \%$ \\
Informative distal & 45 & $96 \%$ & $2 \%$ & $2 \%$ \\
\hline
\end{tabular}

characterised by a palm up position. In declarative distal situations pointing gestures were mostly produced with a palm down position (expressive pointing) or with a palm lateral position (informative pointing).

In imperative situations participants did not produce any pointing gestures with a palm up position. Distal imperative pointing was associated with palm down gestures while proximal imperative pointing was characterised both by palm lateral and palm down positions. However, this distribution relied on a limited number of gestures because participants produced very few whole-hand points in imperative situations.

Symbolic gestures. Participants produced 34 bimanual gestures out of a total of 510 gestures (i.e., approximately $6.7 \%$ ). Both hands always performed identical gestures, so they were included in the analyses. As is presented in Table 3, they produced the target gestures in the majority of cases (target gestures represented between $52.6 \%$ and $97.4 \%$ of total gestures depending on the situation, $M=78.43 \%, S D=19.16 \%$ ). The situations

TABLE 3

Percentages of target symbolic gestures produced in each situation and examples of other gestures produced. Values were rounded to the nearest integer

\begin{tabular}{|c|c|c|c|}
\hline Situation & $\begin{array}{l}\text { Total number of } \\
\text { gestures produced }\end{array}$ & $\begin{array}{l}\text { Percentages of } \\
\text { target gestures }\end{array}$ & $\begin{array}{l}\text { Other gestures } \\
\text { most frequently produced }\end{array}$ \\
\hline a. hush & 78 & $97 \%$ & $\begin{array}{l}\text { "Sleep" gesture (head tilted over onto } \\
\text { hands, palms together) }\end{array}$ \\
\hline b. no & 79 & $71 \%$ & Shaking the head back and forth \\
\hline c. no & 37 & $62 \%$ & $\begin{array}{l}\text { "Stop" gesture (hand extended palm } \\
\text { forward) }\end{array}$ \\
\hline d. hi & 81 & $99 \%$ & "Come here" gesture \\
\hline e. come here & 80 & $98 \%$ & $\begin{array}{l}\text { Movement of the head and the } \\
\text { eyebrows to invite the partner to come }\end{array}$ \\
\hline f. ok, good & 76 & $53 \%$ & Nodding the head \\
\hline g. ok, good & 79 & $70 \%$ & Nodding the head \\
\hline
\end{tabular}


associated with the greater variability in the participants' responses were the "no" and the "OK, good" situations, notably because they could elicit meaningful head movements.

\section{Variations in the form of gestures}

In order to determine whether the variations observed in the form of communicative gestures were explained by variations of contextual features and/or by inter-individual differences in gestural repertoires, we coded for each participant the variations in hand shapes for both pointing gestures and symbolic gestures. The form of pointing gestures was considered as invariant when participants produced the same hand shape (index-finger or wholehand gesture) with the same palm orientation in all the pointing situations. The form of pointing was invariant for 23 participants $(28.4 \%$ of total participants). More precisely, hand shape was invariant for 24 participants $(29.6 \%)$ and palm orientation was invariant for 28 participants $(34.6 \%)$. When palm orientation was constant, hand shape was also constant in the vast majority of cases $(82.1 \%)$. All these participants produced exclusively index-finger pointing with a palm down orientation.

The form of symbolic gestures was considered as invariant when participants produced the same hand shape in the situations designed to elicit the same target gestures (i.e., when they produced the "no" gesture in situations b and c, and "OK, good" gesture in situations $f$ and g). The form of symbolic gestures was invariant for 22 participants $(27.2 \%$ of total participants). Of these 22 participants, 16 (72.7\%) showed variations in hand shape and palm orientation in pointing situations, indicating that in the majority of cases, constancy in the repertoire of symbolic gestures was not associated with constancy in the repertoire of pointing gestures.

\section{Hand preference}

Handedness indices (HI) being skewed towards positive values in humans, hand preference patterns were analysed using non-parametric tests, including Spearman's rank correlations, Wilcoxon signed-rank tests, and MannWhitney $U$ tests. There was no significant effect of gender on hand preference scores, whether it concerned manipulative activities, $Z=0.85$; $n s$, or communicative gestures, $Z=1.30 ; n s$. More precisely, there was no sex difference in the laterality of symbolic gestures, $Z=0.84 ; n s$, but hand preference scores for pointing gestures tended to be lower in males than in females, $Z=1.86 ; p=.063$, in line with several studies showing a greater tendency towards left-handedness in males (for a meta-analysis, see Papadatou-Pastou, Martin, Munafò, \& Jones, 2008). However, when considering each function of pointing separately, we did not find any 
significant effect of gender either for imperative pointing, $Z=0.04 ; n s$, declarative expressive pointing, $Z=1.33$; $n s$, or declarative informative pointing, $Z=0.73$; ns. Therefore we included all the participants in the following analyses.

Communicative gestures. Mean Handedness Index (HI) for communicative gestures was $0.66(S D=0.47)$. A total of 64 participants $(79.01 \%)$ were classified as right-handers, 4 as left-handers (4.94\%), and 13 as ambidextrous $(16.05 \%)$. Mean HIs for pointing gestures $(M=0.65 ; S D=0.52)$ and symbolic gestures $(M=0.68 ; S D=0.48)$ did not differ significantly, $Z=0.83 ; n s(N=81)$. Moreover, hand preference scores for pointing gestures and symbolic gestures were strongly correlated, $r(81)=.60 ; p<.001$.

Manipulative activities. Participants chose the bimanual response for 175 of the questionnaire items, out of a total of $1053(13 * 81)$, which represents about $16.6 \%$. Mean HI for manipulative activities was $0.64(S D=0.50)$. A total of 68 participants $(83.95 \%)$ were classified as right-handers, 5 as lefthanders $(6.17 \%)$, and 8 as ambidextrous $(9.88 \%)$.

Comparison of hand preferences. Mean hand preference indices associated with the different activities are displayed in Figure 1.

First, mean HIs for communicative gestures and manipulative activities did not significantly differ, $Z=0.10 ; n s \quad(N=81)$. The difference was significant neither for pointing gestures, $Z=0.36$; ns $(N=81)$, nor for symbolic gestures, $Z=0.35$; $n s \quad(N=81)$. However, the analysis of the

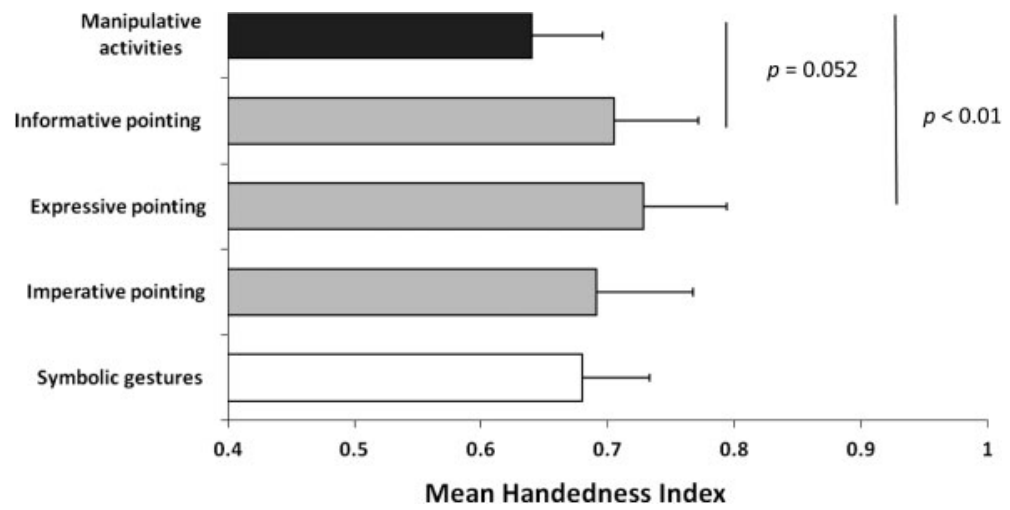

Figure 1. Mean handedness indexes (and standard errors) for manipulative activities (in black), pointing gestures (in grey) and symbolic gestures (in white).

Mean HI varies from 1 to 1 . The positive sign reflects right-hand preference and the absolute values indicate the strength of hand preference within the group of participants.

Significance of Wilcoxon signed-rank tests is indicated by vertical bars. 
different functions of pointing revealed some significant differences with handedness for manipulative activities. Handedness for declarative expressive pointing was stronger than that for manipulative activities, $Z=2.92$; $p<.01(N=81)$, and handedness for declarative informative pointing tended to be stronger than that for manipulative activities, $Z=1.94 ; p=.052$ $(N=72)$. By contrast, there was no significant difference in the strength of handedness between imperative pointing and manipulative activities, $Z=0.35 ; n s(N=55)$.

Spearman's correlations revealed moderate but significant relationships between hand preferences for communicative gestures and manipulative activities, $r(81)=.43 ; p<.001$. The correlations were significant both for pointing gestures, $r(81)=.36 ; p<.001$, and for symbolic gestures, $r(81)=.41$; $p<.001$. In addition, the categorisation of individuals as right-handers, lefthanders, and ambidextrous was congruent for communicative gestures and manipulative activities for 62 participants (76.5\%), including 57 participants (70.4\%) who were right-handed for both activities.

\section{DISCUSSION}

In the present study we investigated the form and the asymmetry of gestures experimentally induced in communicative situations. First we will discuss the results pertaining to the form of pointing gestures and symbolic gestures, and second we will focus on the asymmetry of communicative gestures, compared to non-communicative activities.

\section{Form of pointing gestures}

Pointing can be described as a communicative movement that projects a vector from a body part (Kita, 2003) in order to indicate a referent in the proximal or distal environment. Observational studies have reported numerous variations in the effector used for pointing (hand, mouth, or head) and in the form of manual pointing depending on the communicative context (Kendon \& Versante, 2003; Wilkins, 2003). In young children, pointing gestures produced to get an object (i.e., imperative pointing) are characterised by whole-hand gestures, whereas pointing gestures serving other functions (i.e., declarative expressive and declarative informative pointing) are almost exclusively associated with index-finger gestures (Cochet \& Vauclair, 2010b). It was argued that the different forms of imperative and declarative pointing might be explained by distinct origins, imperative pointing being ritualised from non-communicative reaching actions and declarative pointing being linked from early stages to the development of social and cognitive abilities (e.g., Liszkowski \& Tomasello, 2011). 
In the present study imperative pointing was not characterised by wholehand gestures, suggesting that the use of this gesture in adults is different from its sole instrumental use in infants. Besides, imperative pointing was more frequently associated with index-finger gestures than declarative pointing (both expressive and informative pointing), which may highlight the individual feature of the referent in imperative situations. Requesting an object, or an action on an object, implies referring to something precise and specific, even if the referent is far away from the gesturer. By contrast, the use of declarative gestures is more various because they can indicate specific objects or events, but also directions, landscapes, and referents that are not visible in the immediate environment. Several factors thus come into the picture, which may explain the greater diversity of hand shapes observed in declarative situations compared to the imperative situation. Our results therefore suggest that the form of pointing gestures in declarative situations conveys extra information about the context and about the intention of the gesturer.

First, proximal pointing was more frequently associated with indexfinger gestures than distal pointing in both declarative expressive and declarative informative situations, thus supporting our hypothesis. Hand shape is influenced by the distance between the gesturer and the referent, in relation to the degree of precision and individuation required to identify the referent. Proximal pointing is indeed used for specific and individualised targets-generally small-sized objects - whereas distal pointing is more likely to be used for large referents, including locations, which require less precision (Kendon \& Versante, 2003). This may also explain the low proportion of whole-hand gestures produced in imperative situations, even for distal referents. The individuation of a precise referent which characterises imperative pointing may not fit in with distal situations. As a consequence, the comparison between distal imperative and declarative situations may be limited (the imperative distal situation used in the present study described a referent located a few metres away from the gesturer, whereas in the declarative distal situations, referents were at a distance of several kilometres).

Second, our results showed that the distance between the gesturer and the referent was not the only factor influencing hand shapes. Although we did not expect to observe any difference between expressive and declarative pointing, the former was found to be more frequently associated with indexfinger gestures than the latter, in both proximal and distal situations. Actually, only half of informative gestures were associated with index-finger pointing, suggesting that declarative informative situations may require less precision than declarative expressive situations. Indeed, informative pointing provides the recipients with the precise information they are looking for, in a context where they are thus already attentive to any communicative signal 
that could be helpful. By contrast, it can be hypothesised that expressive gestures were mostly characterised by index-finger pointing because the recipient's attention needs to be directed towards an unsuspected referent, therefore requiring a high level of individuation. Representational gestures have also been reported to be more complex and more precise when they convey information that is new, compared to information that has previously been shared between the gesturer and the addressee (Gerwing \& Bavelas, 2004).

Moreover, the relatively low proportion of index-finger pointing produced in the distal declarative informative situation might be due to the somewhat ambiguous nature of the referent in this situation, which was not directly observable (situation 6). We cannot exclude the possibility that informative distal pointing was used to indicate the direction to the station rather than the station itself, leading to a large proportion of whole-hand gestures.

The present study also revealed some variations in palm orientation depending on the pointing situation. Imperative gestures were never produced with a palm up position, which may emphasise the function of this gesture, whose final goal is to reach and grasp an object. However, palm orientation was only coded for whole-hand gestures (index-finger gestures being almost exclusively produced with a palm down position or with an intermediate position between palm down and palm lateral), which were seldom produced in imperative situations. By contrast, palm up orientations were frequently observed in declarative proximal situations, perhaps as a means of highlighting a referent that is obvious, under the recipient's eyes. In declarative distal situations, palm orientation seemed to reflect specific features of the referent, since palm down gestures were often used to indicate horizontal referents (e.g., the sea, situation 4) whereas palm lateral gestures were used for vertical referents (e.g., straight direction of the station, situation 6).

Our results therefore highlight, in situations where the referent pointed at is in the centre of discourse focus, that both the referent's characteristics and the gesturer's intention can have an effect on hand shapes and palm orientation associated with pointing gestures. Adults' gestures convey thus richer and more complex information than they appear to do in young children, which might be explained by some differences in linguistic skills. Co-speech gestures have indeed been reported to become richer as verbal language complexity increases in the course of development (e.g., Colletta, Pellenq, \& Guidetti, 2010).

However, even though the majority of adult participants used different forms of pointing gestures depending on the situation, more than one quarter of them only produced index-finger pointing with a palm down orientation. This form, which is probably the most widely used in European countries, can be regarded as the conventional form of pointing. It now 
remains to be determined why some individuals only used this form and whether some kind of cultural learning is involved in the association between contextual characteristics and specific forms of pointing.

\section{Form of symbolic gestures}

On the one hand, the present study has shown that within one cultural group - young French adults - a given situation elicited in the majority of cases the same symbolic gesture, especially for the situations a ("hush"), d ("hi"), and e ("come here"). The gestural modality therefore appears to be a natural and efficient way of conveying symbolic meaning, even in adults and even though participants were not given any instruction regarding the communicative modality to use. On the other hand, some situations elicited several different forms of symbolic gestures which fulfilled the same function, thus highlighting the richness of human gestural repertoire. In particular, different hand shapes and different effectors (head, one hand, or both hands) were used to express approval (situations $\mathrm{f}$ and g, "OK, good") and refusal (situations b and c, "no"). This variability may reflect a lower degree of conventionality, namely a less-constrained form-meaning relationship, compared to situations (e.g., situation a, "hush") that elicited almost exclusively one and the same gesture (Parrill, 2008).

Both inter-individual and intra-individual variations were observed in the form of symbolic gestures, in situations supposed to elicit the same target gesture. Although this question deserves further investigation, some of these differences may again be explained by contextual characteristics, including the formality of the context (e.g., Wilkins, 2003). For example, when participants did not produce the target manual gesture to express refusal, most of them shook the head back and forth in a situation requiring some discretion (situation b, "no"), whereas they produced another manual gesture (a "stop" gesture) in a situation that did not require any discretion (situation c, "no").

Another possible explanation of the differences in the form of gestures may lie in the interaction between speech and gestures. Participants were asked to produce what they would naturally do in each situation, i.e., they were not asked to use gestures more than verbal utterances because our goal was to record spontaneous communicative behaviours. As a consequence some differences were observed across participants and across situations in the propensity to produce words, sentences, or other speech sounds simultaneously to gestures (e.g., a few participants produced the "yum" sound to indicate that they are enjoying the cake, situation f), which might have influenced the form of gestures. As indicated above, this issue was not the focus of the present study, but the speech-gesture dynamics may interfere with the production of gestures (e.g., Gullberg, 2011). In particular, the 
simultaneous production of symbolic gestures and corresponding words may have an effect on arm kinematics and voice parameters, compared with conditions under which words or gestures are performed on their own (Barbieri, Buonocore, Dalla Volta, \& Gentilucci, 2009). Moreover, the use of co-speech gestures as a semiotic resource develops with age (Colletta et al., 2010), but the characteristics of gestures have been shown to be influenced by the nature of the discourse in adults. For example, the frequency and the movements of our hands vary depending on whether we are narrating or explaining (e.g., Reig Alamillo, Colletta, \& Guidetti, 2012) and depending on language specificity, leading to cultural variations in the structure of gestures (e.g., Özyürek et al., 2008).

Another potential limitation of the present study is the use of experimental situations to elicit the different gestures. The communicative nature of these situations might be questioned, compared to observational qualitative studies which allow researchers to focus on more natural communicative situations (e.g., Kendon \& Versante, 2003). However, communicative contexts vary widely across individuals in the latter studies, which makes it difficult for researchers to identify the respective contributions of contextual features and individual characteristics to the variations of gestures' form. The study of gestures' asymmetry is also more problematic due to positional biases. We chose to set up experimental situations in order to control these variables more easily and therefore provide a quantitative analysis of communicative gestures in a large sample. Moreover, the participants' responses were not monologues; they were addressed to the experimenter and therefore integrated into a social context (e.g., Bavelas \& Chovil, 2000). Finally, the experimenter did not give any instructions to the participants as to the expected behaviours, but only asked them to produce communicative responses as spontaneously as possible. Thus we can reasonably think that these experimental situations, although different from natural situations, did involve a communicative goal.

\section{Hand preferences}

Results of the present study did not reveal any difference in the degree of hand preference between manipulative activities and communicative gestures, whether it concerned pointing gestures or symbolic gestures. However, consistent with our hypothesis, significant but moderate correlations were shown between the asymmetry of communicative gestures and that of manipulative activities. By contrast, the two types of hand preference are not significantly correlated in young children and the right-sided bias is generally stronger for gestures than for object manipulation (Cochet \& Vauclair, 2010b; Esseily, Jacquet, \& Fagard, 2011; Jacquet et al., 2012). It was argued that some interactions between the control of speech, gestures, and 
manipulative activities gradually develop during childhood, as the left cerebral hemisphere, involved in both communicative skills and fine motor skills required for object manipulation, becomes more and more specialised (Cochet \& Vauclair, 2012). Moreover, the relationship between communicative and non-communicative activities that was observed in the present study may involve the mirror neuron system: results of an fMRI study in adults have shown that the latter was activated to a similar degree by the observation and production of communicative gestures and object-directed movements (Montgomery, Isenberg, \& Haxby, 2007).

However, our results have revealed a stronger correlation between the asymmetry of pointing gestures and that of symbolic gestures than between the asymmetry of communicative gestures and that of non-communicative activities. The distinction between different functions of pointing has also revealed that hand preference for declarative pointing gestures was stronger than that for manipulative activities (this was observed both for expressive and informative pointing, although the difference was associated with a $p$-value of .052 for informative pointing). By contrast, there was no significant difference in the strength of hand preference between imperative pointing and manipulative activities. Therefore, although our results support the existence of intertwined networks controlling communicative gestures and manipulative activities, the distinction observed in young children based on the nature of the activity performed-communicative versus noncommunicative activities - can still be perceived in adults when we study hand preference patterns for different functions of pointing.

A stronger right-sided asymmetry for declarative informative pointing compared to manipulative activities has previously been reported in young children, which was argued to reflect the importance of informative pointing in the development of left-hemispheric specialisation for communicative behaviours (Cochet \& Vauclair, 2010b). More generally, declarative pointing, as opposed to imperative pointing, seems to play a key role in the development of intention-reading abilities and in language acquisition (e.g., Liszkowski et al., 2004, 2006; but see for instance Moore \& Corkum, 1994, and D'Entremont \& Seamans, 2007, for contrasting views on the early forms of psychological understanding associated with infants' gestures). In the present study the stronger degree of hand preference for declarative pointing gestures - but not for imperative pointing gestures or symbolic gestures - compared to manipulative activities therefore might suggest that the declarative function is more strongly involved in the specialisation of the left cerebral hemisphere for communication. Moreover, declarative expressive pointing was regarded as an intermediate gesture between imperative and declarative informative gestures in terms of cognitive and social abilities (Cochet \& Vauclair, 2010b), which may explain why the strong right-sided bias for pointing gestures first involves the declarative informative function 
in children and then all declarative gestures (expressive and informative) in adults. However, these interpretations need to be tested more directly, first with a longitudinal analysis of handedness for different communicative gestures in the course of development, and second with neuroimaging studies providing precise neural correlates of hemispheric specialisation for communication. It would also be beneficial to measure handedness for object manipulation with several methods, namely by proposing unimanual and bimanual tasks along with handedness questionnaire. Moreover, in addition to the function of gestures, it might be useful to take into account other variables that have been shown to influence the asymmetry of co-speech gestures such as the gesturer's emotional state (e.g., Kipp \& Martin, 2009) and again, speech content. For example, the degree of right-hand preference for some co-speech gestures was found to decrease when participants were asked to explain metaphorical linguistic expressions, compared to abstract and concrete expressions (Kita et al., 2007). Thus the contribution of the two cerebral hemispheres in speech and gesture production depends on numerous factors, which need to be considered simultaneously in future studies.

In conclusion, the present study has demonstrated the influence of contextual characteristics (e.g., the function served by the gesture, the distance between the gesturer and the referent pointed at, the formality of the context) on the form of pointing gestures and symbolic gestures. Pointing gestures produced by adults, in addition to directing a partner's attention towards a referent, convey some information that are directly related to the reference situation and may to some extent have acquired a semantic status (Kita, 2009). Moreover, the study of hand preference patterns has shown that declarative pointing gestures, rather than imperative pointing and symbolic gestures, may play a primary role in the development of left-hemisphere specialisation for communicative behaviours. From a phylogenetic perspective our results therefore highlight the relationship between communicative gestures and language, and suggest that the emergence of referential and declarative skills has paved the way for the evolution of a left-lateralised bimodal communication system (Vauclair \& Cochet, 2013). By contrast, several authors have emphasised the importance of pantomimes in the evolution of language (e.g., Gentilucci et al., 2008; Kendon, 2009), the latter gradually evolving towards more symbolic forms of communication (e.g., Corballis, 2010). In line with correlational results of the present study, it can be hypothesised that a close relationship between the use of deictic and symbolic gestures has developed among our Hominin ancestors, leading eventually to the predominance of speech in the human species. Developmental and evolutionary evidence are necessary to investigate further these hypotheses, along with additional observations and experiments in human adults. 
Manuscript received 4 December 2012

Revised manuscript received 13 April 2013

First published online 7 June 2013

\section{REFERENCES}

Barbieri, F., Buonocore, A., Dalla Volta, R., \& Gentilucci, M. (2009). How symbolic gestures and words interact with each other. Brain and Language, 110, 1-11.

Bates, E., Camaioni, L., \& Volterra, V. (1975). The acquisition of performatives prior to speech. Merrill-Palmer Quarterly, 21, 205-226.

Bates, E., O'Connell, B., Vaid, J., Sledge, P., \& Oakes, L. (1986). Language and hand preference in early development. Developmental Neuropsychology, 2, 1-15.

Bavelas, J. B., \& Chovil, N. (2000). Visible acts of meaning: An integrated message model of language use in face-to-face dialogue. Journal of Language and Social Psychology, 19, 163-194.

Butterworth, G., Franco, F., McKenzie, B., Graupner, L., \& Todd, B. (2002). Dynamic aspects of visual event perception and the production of pointing by human infants. British Journal of Developmental Psychology, 20, 1-24.

Camaioni, L. (1997). The emergence of intentional communication in ontogeny, phylogeny and pathology. European Psychologist, 2, 216-225.

Cochet, H., \& Byrne, R. W. (2013). Evolutionary origins of human handedness: Evaluating contrasting hypotheses. Animal Cognition. doi:10.1007/s10071-013-0626-y

Cochet, H., \& Vauclair, J. (2010a). Features of spontaneous pointing gestures in toddlers. Gesture, $10(1), 86-107$.

Cochet, H., \& Vauclair, J. (2010b). Pointing gestures produced by toddlers from 15 to 30 months: Different functions, hand shapes and laterality patterns. Infant Behaviour and Development, 33, 432-442.

Cochet, H., \& Vauclair, J. (2012). Hand preferences in human adults: Noncommunicative actions vs. communicative gestures. Cortex, 48, 1017-1026.

Colletta, J., Pellenq, C., \& Guidetti, M. (2010). Age-related changes in co-speech gesture and narrative: Evidence from French children and adults. Speech Communication, 52(6), 565-576.

Colonnesi, C., Stams, G., Koster, I., \& Noom, M. J. (2010). The relation between pointing and language development: A meta-analysis. Developmental Review, 30(4), 352-366.

Corballis, M. C. (2010). Mirror neurons and the evolution of language. Brain and Language, 112(1), 25-35.

Corbetta, D., \& Thelen, E. (1999). Lateral biases and fluctuations in infants' spontaneous arm movements and reaching. Developmental Psychobiology, 34(4), 237-255.

Crais, E., Day-Douglas, D., \& Cox-Campbell, C. (2004). The intersection of the development of gestures and intentionality. Journal of Speech, Language, and Hearing Research, 47(3), 678-694.

Dalby, J. T., Gibson, D., Grossi, V., \& Schneider, R. D. (1980). Lateralised hand gesture during speech. Journal of Motor Behaviour, 12, 292-297.

D'Entremont, B., \& Seamans, E. (2007). Do infants need social cognition to act socially? An alternative look at infant pointing. Child Development, 78, 723-728.

Enfield, N. J. (2001). 'Lip-pointing': A discussion of form and function with reference to data from Laos. Gesture, 1(2), 185-211.

Enfield, N. J., Kita, S., \& De Ruiter, J. P. (2007). Primary and secondary pragmatic functions of pointing gestures. Journal of Pragmatics, 39(10), 1722-1741.

Esseily, R., Jacquet, A.-Y., \& Fagard, J. (2011). Handedness for grasping objects and pointing and the development of language in 14-month-old infants. Laterality, 16(5), 565-585.

Franco, F., \& Butterworth, G. (1996). Pointing and social awareness: Declaring and requesting in the second year. Journal of Child Language, 23, 307-336. 
Gentilucci, M., \& Dalla Volta, R. (2008). Spoken language and arm gestures are controlled by the same motor control system. The Quarterly Journal of Experimental Psychology, 61, 944-957.

Gentilucci, M., Dalla Volta, R., \& Gianelli, C. (2008). When the hands speak. Journal of Physiology (Paris), 102, 21-30.

Gerwing, J., \& Bavelas, J. B. (2004). Linguistic influences on gesture's form. Gesture, 4, 157-195.

Gullberg, M. (2011). Language-specific encoding of placement events in gestures. In Bohnemeyer, J. \& Pederson, E. (Eds.), Event representation in language and cognition (pp. 166-188). Cambridge, UK: Cambridge University Press.

Jacquet, A.-Y., Esseily, R., Rider, D., \& Fagard, J. (2012). Handedness for grasping objects and declarative pointing: A longitudinal study. Developmental Psychobiology, 54(1), 36-46.

Kendon, A. (2004). Gesture: Visible action as utterance. Cambridge, UK: Cambridge University Press.

Kendon, A. (2009). Language's matrix. Gesture, 9(3), 355-372.

Kendon, A., \& Versante, L. (2003). Pointing by hand in Neapolitan. In S. Kita (Ed.), Pointing: Where language, culture, and cognition meet (pp. 109-137). Mahwah, NJ: Erlbaum.

Kimura, D. (1973). Manual activity during speaking: I. Right-handers. Neuropsychologia, 11, $45-50$.

Kita, S. (2003). Pointing: A foundational building block of human communication. In S. Kita (Ed.), Pointing: Where language, culture, and cognition meet (pp. 1-8). Mahwah, NJ: Erlbaum.

Kita, S. (2009). Cross-cultural variation of speech-accompanying gesture: A review. Language and Cognitive Processses, 24(2), 145-167.

Kita, S., Condappa, O., \& Mohr, C. (2007). Metaphor explanation attenuates the right-hand preference for depictive co-speech gestures that imitate actions. Brain and Language, 101, 185-197.

Kipp, M., \& Martin, J. C. (2009). Gesture and emotion: Can basic gestural form features discriminate emotions? Proceedings of the 3rd international workshops on Affective Computing and Intelligent Interaction (ACII 2009). Amsterdam: LNCS, Springer.

Liszkowski, U. (2008). Before L1: A differentiated perspective on infant gestures. Gesture, 8(2), 180-196.

Liszkowski, U., Carpenter, M., Henning, A., Striano, T., \& Tomasello, M. (2004). Twelve-montholds point to share attention and interest. Developmental Science, 7, 297-307.

Liszkowski, U., Carpenter, M., Striano, T., \& Tomasello, M. (2006). Twelve- and 18-month-olds point to provide information for others. Journal of Cognition and Development, 7, 173-187.

Liszkowski, U., \& Tomasello, M. (2011). Individual differences in social, cognitive, and morphological aspects of infant pointing. Cognitive Development, 26, 16-29.

McNeill, D. (1992). Hand and mind: What gestures reveal about thought. Chicago, IL: University of Chicago Press.

McNeill, D. (2000). Language and gesture: Window into thought and action. Cambridge, UK: Cambridge University Press.

McNeill, D. (2005). Gesture and thought. Chicago, IL: University of Chicago Press.

Montgomery, K. J., Isenberg, N., \& Haxby, J. V. (2007). Communicative hand gestures and objectdirected hand movements activated the mirror neuron system. Social Cognitive and Affective Neuroscience, 2, 114-122.

Moore, C., \& Corkum, V. (1994). Social understanding at the end of the first year of life. Developmental Review, 14, 349-372.

Morris, D., Collett, P., Marsh, P., \& O’Shaughnessy, M. (1979). Gestures: Their origins and distribution. London: Jonathan Cape.

Oldfield, R. C. (1971). The assessment and analysis of handedness: The Edinburgh inventory. Neuropsychologia, 9, 97-113.

Özyürek, A., Kita, S., Allen, S., Brown, A., Furman, R., \& Ishizuka, T. (2008). Development of cross-linguistic variation in speech and gesture: Motion events in English and Turkish. Developmental Psychology, 44, 1040-1054. 
Papadatou-Pastou, M., Martin, M., Munafò, M.R., \& Jones, G.V. (2008). Sex differences in lefthandedness: A meta-analysis of 144 studies. Psychological Bulletin, 134(5), 677-699.

Parrill, F. (2008). Form, meaning and convention: An experimental examination of metaphoric gestures. In A. Cienki \& C. Müller (Eds.), Metaphor and gesture (pp. 195-217). Amsterdam: John Benjamins.

Reig Alamillo, A., Colletta, J. M., \& Guidetti, M. (2012). Gesture and language in narratives and explanations: The effects of age and communicative activity on late multimodal discourse development. Journal of Child Language, 40(3), 511-538. doi:10.1017/S0305000912000062

Saucier, D. M., \& Elias, L. J. (2001). Lateral and sex differences in manual gesture during conversation. Laterality, 6, 239-245.

Tomasello, M., Carpenter, M., \& Liszkowski, U. (2007). A new look at infant pointing. Child Development, 78, 705-722.

Vauclair, J., \& Cochet, H. (2012). Hand preference for pointing and language development in toddlers. Developmental Psychobiology. doi:10.1002/dev.21073

Vauclair, J., \& Cochet, H. (2013). Ontogeny and phylogeny of communicative gestures, speechgestures relationships and left hemisphere specialisation for language. In R. Botha \& M. Everaert (Eds.), Oxford studies in the evolution of language: The evolutionary emergence of human language. Oxford, UK: Oxford University Press.

Vauclair, J., \& Imbault, J. (2009). Relationships between manual preferences for object manipulation and pointing gestures in infants and toddlers. Developmental Science, 12, 1060-1069.

Wilkins, D. (2003). Why pointing with the index finger is not a universal (in sociocultural and semiotic terms). In S. Kita (Ed.), Pointing: Where language, culture, and cognition meet (pp. 171-215). Mahwah, NJ: Erlbaum.

Xu, J., Gannon, P. J., Emmorey, K., Smith, J. F., \& Braun, A. R. (2009). Symbolic gestures and spoken language are processed by a common neural system. Proceedings of the National Academy of Sciences (USA), 106(49), 20664-20669. 


\section{APPENDIX \\ Description of the 13 communicative situations eliciting pointing gestures (situations 1-6) and symbolic gestures (situations a-g).}

\begin{tabular}{ll} 
Gesture & \multicolumn{1}{c}{ Description } \\
\hline $\begin{array}{l}\text { 1. Proximal } \\
\text { imperative } \\
\text { pointing }\end{array}$ & $\begin{array}{l}\text { The arm and the hand, either the } \\
\text { whole hand or just the index finger, } \\
\text { are extended towards a referent. The } \\
\text { target referent is close to the } \\
\text { gesturer (within } 2 \text { metres). }\end{array}$
\end{tabular}

2. Distal imperative pointing

3. Proximal expressive pointing

4. Distal expressive pointing

5. Proximal informative pointing

6. Distal informative pointing

a. "hush"

b. "no"
The arm and the hand, either the whole hand or just the index finger, are extended towards a referent. The target referent is far from the gesturer (more than 2 metres away). The arm and the hand, either the whole hand or just the index finger, are extended towards a referent. The target referent is close to the gesturer (within 2 metres).

The arm and the hand, either the whole hand or just the index finger, are extended towards a referent. The target referent is far from the gesturer (more than 2 metres away). The arm and the hand, either the whole hand or just the index finger, are extended towards a referent. The target referent is close to the gesturer (within 2 metres).

\section{The arm and the hand, either the} whole hand or just the index finger, are extended towards a referent. The target referent is far from the gesturer (more than 2 metres away). The index finger is placed over the mouth.

The whole hand or the index finger is raised and oscillates from right to left.
Scenarios

You are having lunch with your family, there are a lot of people around the table and the atmosphere is quite noisy. You want your grandfather, who has some hearing troubles, to pass you the salt which is on the table in front of him. How do you ask him?

You are in a decoration shop, looking for a new lamp for your apartment. You want the salesman to give you a lamp that is placed too high for you. How do you ask him?

You are glancing through a gardening magazine. You want your friend to look at the flowers you would like to plant in your garden. How do you show them to your friend?

You are having a walk with a friend and you want him to look at the sea on the horizon. How do you show him?

Your friend is looking after his keys that he has just lost. They are under your eyes, on the shelf in front of you. How do you show them to your friend?

You are in the street. A passer-by is asking you about the direction of the train station, which is at a few kilometres straight ahead. How do you indicate it?

It is naptime at the day-care centre, but a child is not asleep. You want this child to stay silent so that he does not wake the other children. How do you make yourself understood?

A friend is playing cards with another person. You want to help your friend by looking at his opponent's cards. You place yourself behind the latter so that he can't see you and you want to tell your friend not to play on the next turn. How do you do? 
APPENDIX

(Continued)

\begin{tabular}{lll}
\hline Gesture & \multicolumn{1}{c}{ Description } & \multicolumn{1}{c}{ Scenarios } \\
\hline c. "no" & $\begin{array}{l}\text { The whole hand or the index finger } \\
\text { is raised and oscillates from right to } \\
\text { left. }\end{array}$ & $\begin{array}{l}\text { You are babysitting your nephew who is } 5 \\
\text { years old. During snack time, he slowly } \\
\text { and intentionally pushes the biscuit } \\
\text { packet near the edge of the table, as if he } \\
\text { wanted to make it fall to the ground. How } \\
\text { do you indicate him not to do it? }\end{array}$ \\
d. "hi", "bye" & $\begin{array}{l}\text { The hand is raised towards the } \\
\text { recipient and possibly moves to and } \\
\text { fro. }\end{array}$ & $\begin{array}{l}\text { You are in a demonstration, it is very } \\
\text { noisy. You have just seen a friend in the } \\
\text { middle of the crowd. How do you greet }\end{array}$ \\
him in spite of the distance?
\end{tabular}

